\title{
An open-label, flexible-dose study of paliperidone extended-release in Chinese patients with first-onset psychosis
}

This article was published in the following Dove Press journal:

Neuropsychiatric Disease and Treatment

6 January 2015

Number of times this article has been viewed

\author{
TianMei $\mathrm{Si}^{1}$ \\ QingRong Tan ${ }^{2}$ \\ KeRang Zhang ${ }^{3}$ \\ Yang Wang ${ }^{4}$ \\ Qing Rui ${ }^{4}$
}

'Peking University Institute of Mental Health, Key Laboratory of Mental Health, Ministry of Health (Peking University), Beijing, ${ }^{2}$ Fourth Military Medical University, First Hospital, Xi'an, ${ }^{3}$ Shanxi Medical University, First Hospital, Shanxi, ${ }^{4}$ Janssen Research and Development, Beijing, People's Republic of China
Correspondence: TianMei Si Peking University Institute of Mental Health, Huayuanbeilu 5I\#, Haidian District, Beijing I00191, People's Republic of China $\mathrm{Tel}+861082801948$

Email si.tian-mei@I63.com
Background: Antipsychotic medications facilitate the improvement of psychotic symptoms in patients with first-episode psychosis. Paliperidone extended-release (pali-ER), an atypical antipsychotic, was assessed for efficacy and safety in Chinese patients with first-episode psychosis.

Methods: In this 8-week, open-label, single-arm, multicenter study, patients with firstepisode psychosis (Diagnostic and Statistical Manual of Mental Disorders, Fourth Edition criteria) and a Positive and Negative Syndrome Scale (PANSS) total score $\geq 70$ were treated with flexible-dose pali-ER tablets (3-12 $\mathrm{mg}$ /day). The primary efficacy endpoint was the percentage of patients with an increase of $\geq 8$ points in Personal and Social Performance (PSP) score from baseline to day 56 (8 weeks). Secondary endpoints included reduction in PANSS total score, improvement in Clinical Global Impression-Severity score, PSP score, Subjective Well-being under Neuroleptics Scale score, and relationship between duration of untreated psychosis and PANSS or PSP. Incidences of treatment-emergent adverse events were used to evaluate safety.

Results: Overall, 283 of 294 patients (96\%) achieved a $\geq 8$-point increase in PSP (primary endpoint, analysis set). For the secondary efficacy endpoints, 284/306 patients (93\%) had $\mathrm{a} \geq 30 \%$ reduction in PANSS total score; $266 / 306$ patients (87\%) achieved a $\leq 3$ Clinical Global Impression-Severity scale score, and $218 / 294$ patients (74\%) had a PSP score $\geq 71$. The Subjective Well-being under Neuroleptics Scale score was improved from a baseline mean of 72.7 to 94.7 at endpoint. There was a negative correlation between duration of untreated psychosis and posttreatment PSP score and a positive correlation with posttreatment PANSS total score. The most common treatment-emergent adverse events were extrapyramidal symptoms (12\%), and agitation, somnolence, and xerostomia (4\% each).

Conclusion: An 8-week, flexible-dose (3-12 mg/day) treatment with pali-ER resulted in significant improvements in psychotic symptoms and social functioning in Chinese patients with first-episode psychosis and was generally tolerable.

Keywords: paliperidone, first-episode psychosis, Personal and Social Performance score

\section{Introduction}

A high prevalence of suicidal tendency is noted in the early course of schizophrenia, ${ }^{1,2}$ a major disease accounting for $55 \%$ of all psychotic disorders in the adult Chinese population. ${ }^{3}$ Antipsychotic medications aid in the improvement of psychotic symptoms in patients with first-episode psychosis (FEP). ${ }^{4}$ However, substantial clinical data indicate that lack of treatment and medication nonadherence increases the risk of clinical relapse in patients with FEP. ${ }^{5,6}$ Recurrent psychotic episodes increase treatment costs and the risk of developing persistent psychotic symptoms and social dysfunction. Further, relapse in young patients affects their long-term psychosocial 
development, and chronic depression and suicidal tendency are major causes for concern. ${ }^{5,7}$ Active, well-managed antipsychotic treatment of patients with FEP is crucial because they are usually younger, with a shorter duration of psychosis and a greater likelihood of controlling their symptoms, and consequently improving their social function is greater as compared with other types of psychosis.

Paliperidone extended-release (pali-ER), marketed as Invega $^{\circledR}$, is a once-daily atypical antipsychotic approved in many countries, including the People's Republic of China, for the treatment of schizophrenia in adults. Several placebo-controlled clinical studies conducted in non-Chinese populations have demonstrated an improvement in positive and negative symptoms with pali-ER treatment in patients with schizophrenia. ${ }^{8,9}$ The safety and efficacy of pali-ER as compared with other antipsychotic drugs has also been evaluated in Chinese patients with FEP. ${ }^{10,11}$ The role of pali-ER in FEP has been evaluated in smaller studies that focused more on remission of symptoms and less on social functioning and subjective well-being in response to the treatment. Also, they did not consider factors that may influence the progression and prognosis of schizophrenia, such as duration of untreated psychosis. ${ }^{12,13}$

The current study examined the efficacy (including symptom control and functional improvement) of pali-ER in Chinese patients with FEP and attempted to determine the relationship between duration of untreated psychosis and functional improvement of FEP in the acute phase. The safety and tolerability of pali-ER was also evaluated.

\section{Materials and methods Study participants}

Chinese male and female inpatients and outpatients, aged 18-65 years and diagnosed with FEP, who met Diagnostic and Statistical Manual of Mental Disorders, Fourth Edition criteria, and were in the acute phase of the illness with a Positive and Negative Syndrome Scale (PANSS) total score $\geq 70$ were enrolled. FEP was defined as the first onset of psychosis with no treatment, with treatment at a less than effective dosage or for less than 4 successive weeks, or symptoms that were alleviated but improvement had been for less than 6 months.

Patients with a diagnosis of substance dependence or a disorder that could affect the absorption, metabolism, or excretion of the drug were excluded from the study. A history of neuroleptic malignant syndrome or tardive dyskinesia, a positive urine pregnancy test, concomitant medication that could prolong the QT interval, and hypersensitivity to pali-ER or risperidone were also exclusion criteria.
The independent ethics committee or institutional review board at each study site approved the protocol, and the study was conducted in accordance with the ethical principles of the Declaration of Helsinki and consistent with Good Clinical Practices and applicable regulatory requirements. All patients provided their written informed consent before enrollment.

\section{Study design}

This flexible-dose, open-label, single-arm, multicenter, prospective study was conducted from January 22, 2010 to September 13, 2010 at nine sites in the People's Republic of China. The study consisted of two phases, ie, a screening/baseline phase and an 8-week, open-label treatment phase.

All patients received flexibly dosed (3-12 mg) pali-ER tablets once daily. The pali-ER tablets were supplied in blister packages of $3 \mathrm{mg}$ and $6 \mathrm{mg}$, and were administered at doses of $3,6,9$, or $12 \mathrm{mg}$. The recommended starting dose was $6 \mathrm{mg}$. However, a starting dose of $3 \mathrm{mg}$ was acceptable for patients with drug intolerance. The target dose was reached and dose adjustments were made using increments of $3 \mathrm{mg}$ at the investigator's discretion based on the patient's clinical history and symptoms. At each visit, investigators confirmed the actual dose taken with both the patient and their caregiver. The new prescribed dose was also recorded.

Pali-ER tablets were administered once daily in the morning. Patients were directed to consume each tablet either in the fasting state or combined with breakfast consistently, and not to alternate between fasting and post-meal administration.

\section{Concomitant medications}

Following local treatment guidelines, ${ }^{14}$ use of mood stabilizers, antidepressants, and other antipsychotic drugs was not recommended. Benzodiazepines were permissible, if required, for anxiety or/and insomnia. Previous medications for treatment of FEP were to be discontinued within 2 weeks before administration of the first dose of the study drug.

\section{Efficacy assessments}

The primary efficacy endpoint was the percentage of patients with an increase of $\geq 8$ points on the Personal and Social Performance (PSP) scale from baseline to day 56 (end of 8 weeks). Scores on the PSP scale and its four domains (socially useful activities, personal and social relationships, self-care, and disturbing and aggressive behavior) were assessed at baseline, day 28 , and day 56 , and the 
patients were grouped into six categories (absent, mild, manifest, marked, severe, and very severe) in each of the four domains.

Secondary efficacy assessments included the percentage of patients with a $\geq 30 \%$ reduction in PANSS total score and a Clinical Global Impression-Severity (CGI-S) scale score $\leq 3$ from baseline to day 56 (week 8), the percentage of patients with a PSP scale score $\geq 71$ at day 56 , and the relationship between duration of untreated psychosis and PANSS or PSP at day 56. The changes in PANSS total scores, PANSS Marder factor scores, CGI-S scores, and Subjective Well-being under Neuroleptics Scale (SWN) scores from baseline to endpoint were also assessed. Additionally, the percentages of patients grouped by three levels of PSP total scores (1-30 points, $31-70$ points and $71-100$ points) were also determined.

\section{Safety assessments}

Treatment-emergent adverse events (TEAEs), clinical laboratory parameters, electrocardiograms, weight, vital signs, physical examination, and the Extrapyramidal Symptom Rating Scale were used to evaluate safety.

\section{Statistical analysis}

The sample size was determined based on the results of a previous similar study wherein an increase in PSP score of $\geq 8$ points occurred in $80 \%$ of patients after treatment with pali-ER. ${ }^{12}$ A total of 246 patients was considered sufficient to provide a two-sided $95 \%$ confidence interval (CI) for the sample responder rate with a precision equal to 0.1 . Assuming a 10\%-20\% dropout rate, it was planned to enroll 300 patients.

The analysis set for the primary efficacy endpoints represented the full analysis set (FAS), which included all patients who received at least one dose of pali-ER and had at least one post-baseline efficacy evaluation. Primary and secondary endpoints were analyzed descriptively.

Depending on whether the changes between baseline and endpoint met a Gaussian distribution, the Student's $t$-test or paired Wilcoxon signed rank test was used. The qualitative data were analyzed using the $\chi^{2}$ test or Fisher's Exact test. The statistical analysis was performed using SAS version 9.1 for Windows software (SAS Institute Inc, Cary, NC, USA).

All safety assessments were analyzed descriptively and were performed on the safety analysis set, which included all patients who received at least one dose of pali-ER and provided at least one safety assessment data point (patients who have provided at least one time safety assessment at scheduled visit).

\section{Results}

\section{Study participants}

Of the 313 enrolled Chinese patients, 308 (98.4\%) were included in the safety set and 306 (97.76\%) (Table S1) were included in the FAS (Table S1). A total of 269/308 patients $(87.3 \%)$ received 8 weeks of treatment without any major protocol violation (Figure 1).

The proportion of men and women was similar $(50 \%)$ in the FAS, with a mean ( \pm standard deviation) age of $30.00 \pm 11.21$ years and a body mass index of $21.61 \pm 2.9 \mathrm{~kg} / \mathrm{m}^{2}$. The majority $(69.3 \%)$ of patients were diagnosed with paranoid schizophrenia, and had suffered from psychosis for a mean duration of $1.5 \pm 3.20$ years. The mean baseline PSP score was $41.39 \pm 12.21$ and the PANSS total score was 95.34 \pm 18.50 . The median duration of lack of treatment was 139 days (Table 1).

\section{Extent of exposure}

The actual mean daily dose of pali-ER taken was $3.65 \pm 2.14 \mathrm{mg}$ at baseline and $6.53 \pm 1.82 \mathrm{mg}$ at day $56 \pm 3$ (Figure 2A). However, the prescribed mean doses were $4.38 \pm 1.50 \mathrm{mg}$ at baseline and $6.42 \pm 1.87 \mathrm{mg}$ at day $56 \pm 3$ (Figure 2B).

\section{Primary efficacy}

In the FAS, after 8 weeks of treatment with pali-ER, $283 / 294$ patients $(96.26 \%)$ achieved a clinically relevant improvement in the PSP score ( $\geq 8$ points increase from

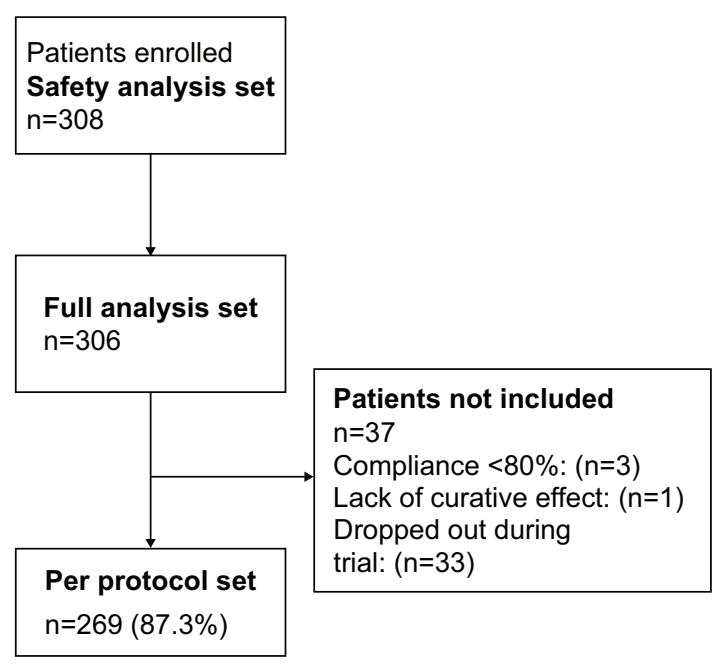

Figure I Patient disposition.

Notes: Full analysis set, patients who received at least one dose of pali-ER with at least one efficacy evaluation; per protocol set, patients who received 8 weeks of treatment and had no protocol violation; safety set, all patients who took at least one dose of pali-ER and provided at least one set of safety assessment data. Abbreviation: pali-ER, paliperidone extended-release. 
Table I Demographic and baseline characteristics (full analysis set)

\begin{tabular}{|c|c|}
\hline Items & Statistics \\
\hline Age (years), mean (SD) & $30.0(11.2)$ \\
\hline \multicolumn{2}{|l|}{ Sex, n (\%) } \\
\hline Men & $153(50 \%)$ \\
\hline Women & 153 (50\%) \\
\hline Weight (kg), mean (SD) & $60(10.4)$ \\
\hline Height $(\mathrm{cm})$, mean $(\mathrm{SD})$ & $166.3(6.9)$ \\
\hline BMI $\left(\mathrm{kg} / \mathrm{m}^{2}\right)$, mean $(\mathrm{SD})$ & $21.6(2.9)$ \\
\hline \multicolumn{2}{|l|}{ BMI category, n (\%) } \\
\hline$<18$ & $29(9.5)$ \\
\hline $18-25$ & $238(78.0)$ \\
\hline$>25$ & $38(12.5)$ \\
\hline \multicolumn{2}{|c|}{ Categories of first-episode psychosis } \\
\hline Total (missing) & $306(0)$ \\
\hline Schizophreniform disorder & $32(10.5)$ \\
\hline Schizoaffective disorder & $4(1.3)$ \\
\hline Schizophrenia & $270(88.2)$ \\
\hline Total (missing) & $270(0)$ \\
\hline Paranoid type & $187(69.3)$ \\
\hline Disorganized type & I (0.4) \\
\hline Catatonic type & $\mathrm{I}(0.4)$ \\
\hline Simple type & $5(1.9)$ \\
\hline Undifferentiated type & $76(28.2)$ \\
\hline \multicolumn{2}{|l|}{ Duration of psychosis (years)* } \\
\hline $\mathrm{n}$ (missing) & $306(0)$ \\
\hline Mean (SD) & $1.5(3.2)$ \\
\hline Median & 0.4 \\
\hline Range & $0.00-30.05$ \\
\hline $95 \% \mathrm{Cl}$ & $1.1-1.8$ \\
\hline \multicolumn{2}{|c|}{ Duration of untreated period (days) } \\
\hline $\mathrm{n}$ (missing) & $301(5)$ \\
\hline Median & 139.0 \\
\hline Range & $2.0-10,976.0$ \\
\hline $95 \% \mathrm{Cl}$ & $310.8-554.1$ \\
\hline
\end{tabular}

Note: *Duration of psychosis $=($ date of signing informed consent form - date of first onset of psychosis +I)/365.25.

Abbreviations: $\mathrm{BMI}$, body mass index; $\mathrm{Cl}$, confidence interval; SD, standard deviation.

baseline to endpoint). At week 8, mean PSP scores had increased from baseline by 34.30 points (Figure 3). Patients' ability to function, as assessed by PSP scales, also improved from baseline to endpoint (Figure 4). The severity level of the four domains of the PSP scale was also significantly improved $(P<0.001)$ at endpoint (Figure 5).

\section{Secondary efficacy}

Psychiatric symptoms were improved after 8 weeks of treatment with pali-ER: $284 / 306$ patients $(92.8 \%)$ had a $\geq 30 \%$ reduction in PANSS total score from baseline to endpoint. The PANSS total score improved significantly $(P<0.0001)$ over time, with a mean change of $51.8 \pm 21.6$ at endpoint (Figure 6). The mean PANSS subscale scores also improved significantly $(P<0.0001)$, with the most improvement seen in symptoms of psychopathology (24.15 \pm 11.80$)$, followed by positive symptoms $(16.76 \pm 6.63)$ and negative symptoms (10.89 \pm 7.18$)$ from baseline to endpoint. PANSS Marder factor scores improved significantly $(P<0.0001)$ after 8 weeks of treatment with pali-ER, with the greatest improvement occurring in positive symptoms (Figure 7).

At endpoint, 218 patients $(74.2 \%, 95 \%$ CI 69.15-79.15) had a PSP score $\geq 71$, indicating substantial clinical improvement, and 266 patients (86.9\%, 95\% CI 83.15-90.71) achieved moderate improvement in their CGI-S score. The mean change in PANSS total scores was 51.80 \pm 21.60 and in the CGI-S score was 3.20 \pm 1.21 .

Mean SWN scores increased (21.97 \pm 20.38$)$ significantly $(P<0.0001)$ from baseline to endpoint (Figure 8$)$. The proportion of patients who felt discomfort decreased from $72.8 \%$ at baseline to $20.9 \%$ at week 8 , and the proportion of patients who felt comfortable increased from $8.5 \%$ to $56.9 \%$ (Figure 9).

There was a negative correlation between duration of untreated psychosis and posttreatment PSP score ( $r=-0.2019, P=0.0006$ ), and a positive correlation with posttreatment PANSS total score $(r=0.1952, P=0.0007)$, indicating that a shorter untreated period is associated with better social functioning and a more favorable prognosis.
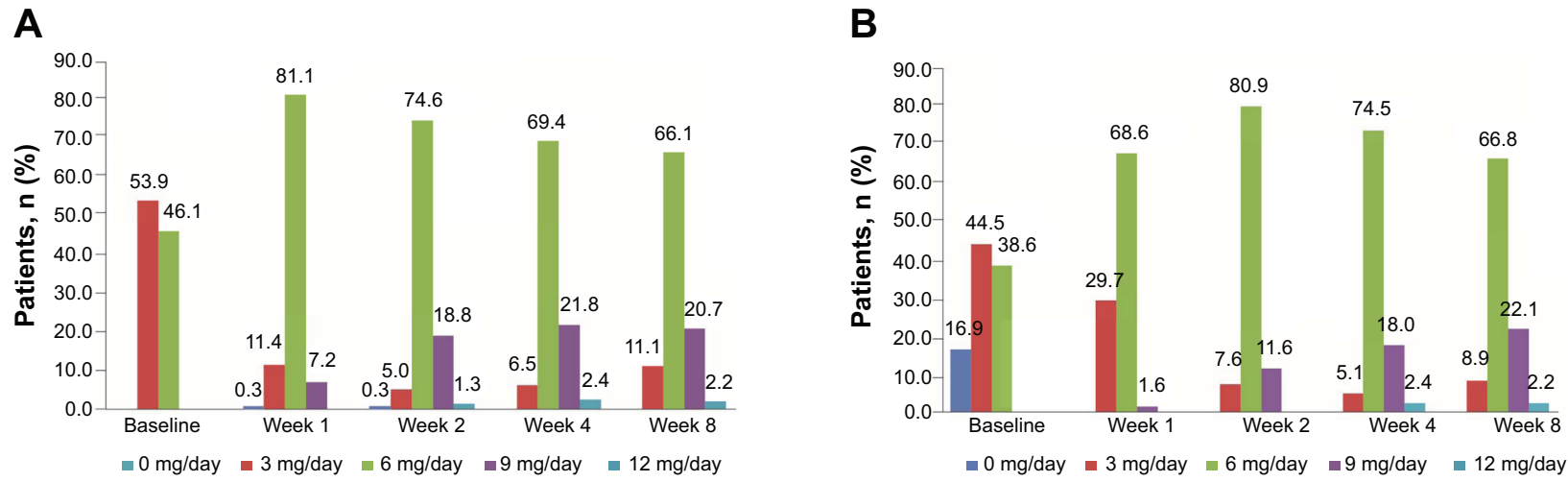

Figure 2 (A) Number of patients with prescribed doses as planned at the start of the visits. (B) Number of patients with actual doses administered at each visit. 


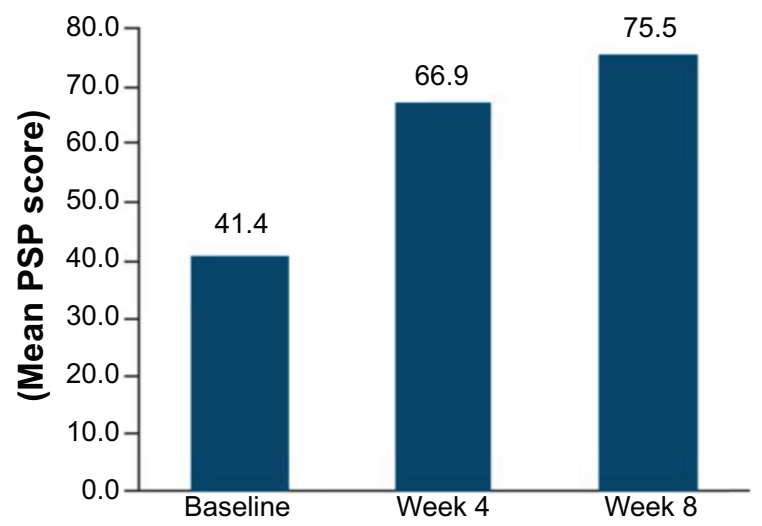

Figure 3 Mean PSP score at each visit.

Abbreviation: PSP, personal and social performance.

\section{Safety results}

Ninety-five (30.84\%) of 308 patients in the safety analysis set experienced at least one TEAE. The most common TEAEs (incidence $>3 \%$ ) were extrapyramidal symptoms (12.01\%), agitation and somnolence (4.22\% each), xerostomia and weakness (3.57\% each), and bradykinesia (3.25\%, Table 2$)$. No deaths occurred during the study. TEAEs leading to permanent discontinuation of the study drug (leukopenia, excitement, and extrapyramidal symptoms) were noted in three $(0.97 \%)$ patients. Three patients $(0.97 \%)$ experienced serious TEAEs, ie, depression, agitation, and extrapyramidal symptoms. Mild or moderate TEAEs related to hyperprolactinemia were reported in six $(1.95 \%)$ patients. Mean patient weight increased significantly $(P<0.0001)$ from baseline $(59.92 \pm 10.36 \mathrm{~kg})$ to the 8 -week endpoint $(61.74 \pm 10.19 \mathrm{~kg})$. The mean extrapyramidal symptom score was increased from baseline $(0.25 \pm 1.15)$ to endpoint $(0.37 \pm 1.10)$. There were no significant increases in fasting glucose. No clinically relevant changes were observed in vital signs, electrocardiographic recordings, or other clinical laboratory parameters.

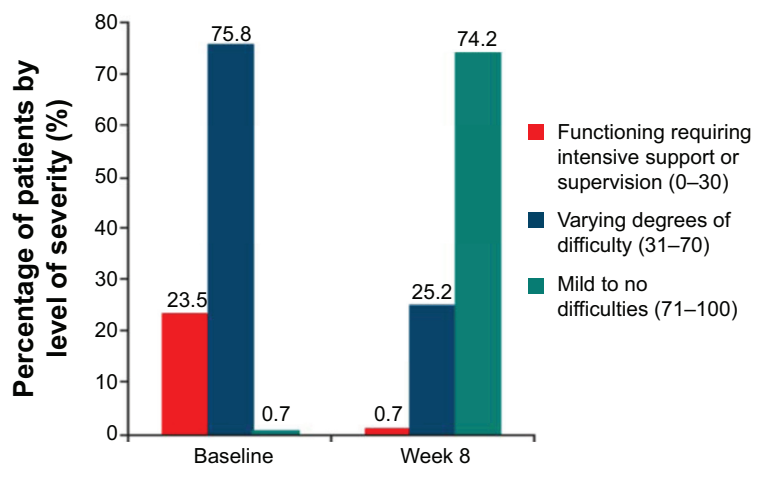

Figure 4 Distribution of patients by severity level of PSP score at baseline and endpoint.

Abbreviation: PSP, personal and social performance.

\section{Discussion}

Treatment with flexible-dose (3-12 mg/day) pali-ER tablets resulted in overall improvement in social functioning in patients with FEP, as indicated by improved PSP scores $(\geq 8)$ at the 8-week endpoint of this open-label study. By week 4, the PSP total score had increased by over 20 points and continued to improve to the end of the trial, suggesting that social and personal functioning can improve rapidly with treatment. Further, improvement in socially useful activity and personal and social relationship domains of the PSP score also occurred following a short period of pali-ER treatment.

A PANSS total score decrease of $\geq 30 \%$ from baseline was used as a criterion for clinically significant improvement, based on previously published data. A larger proportion of patients $(92.81 \%)$ achieved this criterion than in a previous placebo-controlled, 6-week study in a white population $(50 \%)$ or in an open-label, 8-week study in a similar population $(82.6 \%))^{8,12}$ This difference may be a result of the more responsive, relatively naïve patients enrolled in the current study, who had been treated previously with other antipsychotics at an insufficient dose or for an inadequate duration.

Psychiatric symptoms, as assessed by the PANSS subscale scores and Marder factor scores, also improved, which is consistent with previous studies conducted in similar populations. ${ }^{12,13}$ However, the mean daily dose of pali-ER actually taken at week $8(6.5 \pm 1.8 \mathrm{mg})$ was less than in a previous study $(7.9 \pm 2.2 \mathrm{mg})$ also conducted in a Chinese population. ${ }^{12}$

Patients with FEP are more sensitive to antipsychotic drugs and show a rapid improvement in positive psychotic symptoms. ${ }^{4}$ Thus, the optimum dose for individual patients and possible adverse effects of a drug may vary in clinical practice. The open-label design with flexible-dose treatment in this study simulates the clinical practice currently followed in hospital settings.

In this study, the number of patients initially prescribed a $3 \mathrm{mg}$ dose of pali-ER decreased from baseline (53\%) to week $1(11 \%)$. This may have resulted from the need for higher doses when treating more pronounced symptoms. However, it could also reflect the fact that pali-ER had just been launched in the People's Republic of China when this study was conducted. Consequently, Chinese psychiatrists may have been reluctant to start with the higher $6 \mathrm{mg}$ dose as recommended in the labeling, perhaps due to apprehension about safety and tolerability, and thus opted to initiate dosing at $3 \mathrm{mg}$. Currently, $6 \mathrm{mg}$ is generally prescribed as a starting dose because acceptable tolerability of pali-ER has been 


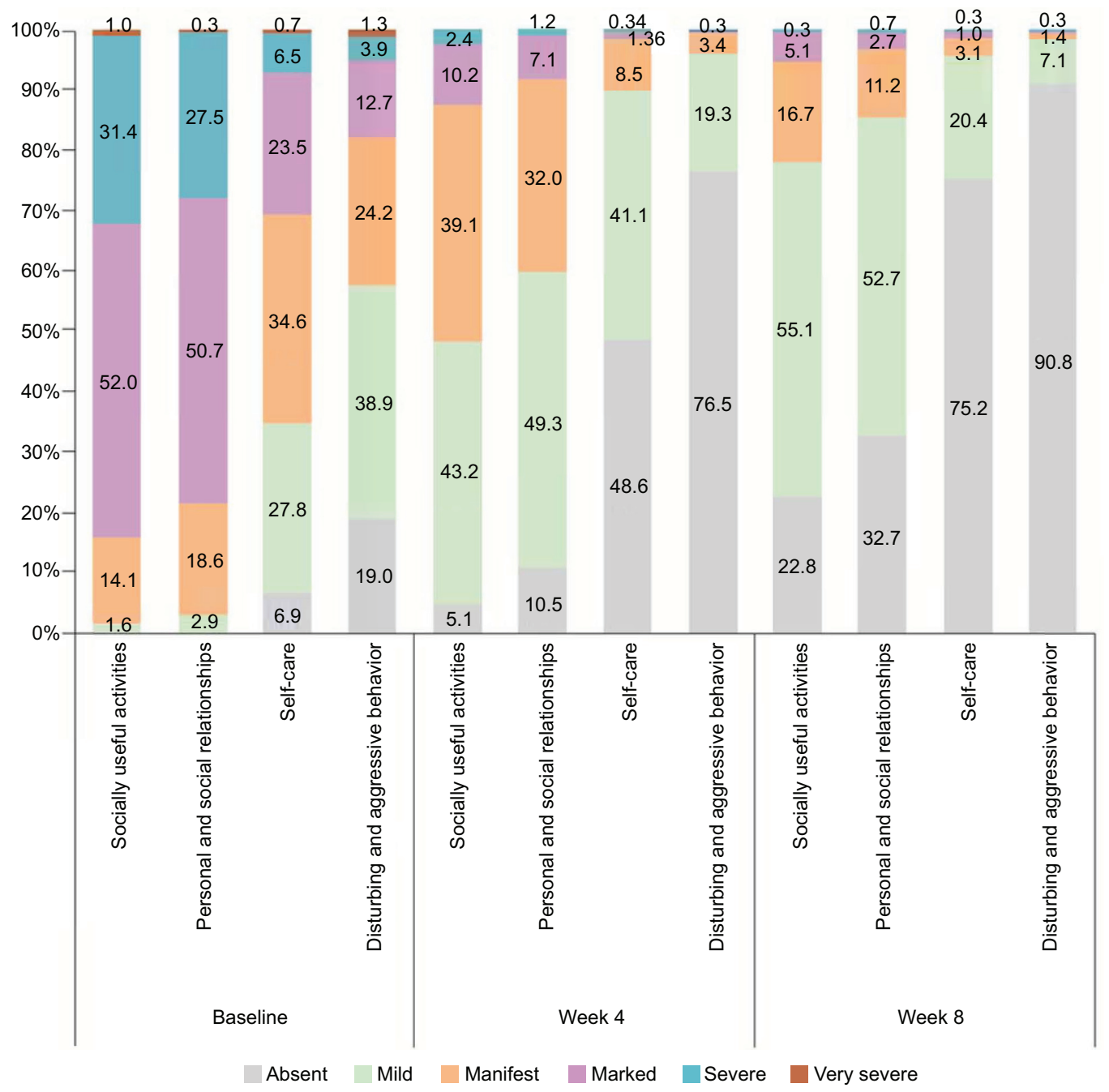

Figure $\mathbf{5}$ Changes in impairment levels as assessed by the four domains of the PSP scale over time. Abbreviation: PSP, personal and social performance.

demonstrated in this and several similar placebo-controlled studies, ${ }^{8}$ and has been seen in routine clinical practice.

In order to measure self-reported subjective well-being, the SWN-20 score ${ }^{15}$ has been widely used as it is one of the important outcome variables of antipsychotic treatment.

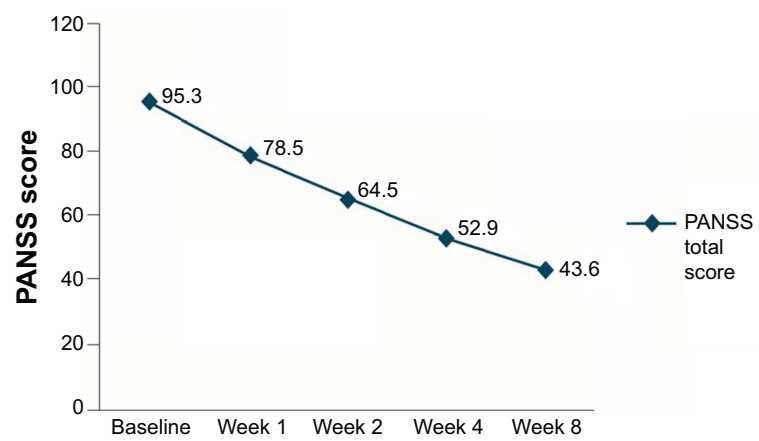

Figure 6 Mean PANSS total score at each visit. Abbreviation: PANSS, Positive and Negative Syndrome Scale.
The Chinese version of the SWN-20 was validated in 2003, with good internal consistency (Cronbach's $\alpha=0.839$ ) and demonstrating a correlation with the global well-being schedule (0.652), Self-Rating Anxiety Scale score (-0.641), and Self-Rating Depression Scale score $(-0.674)$. Two cutoff

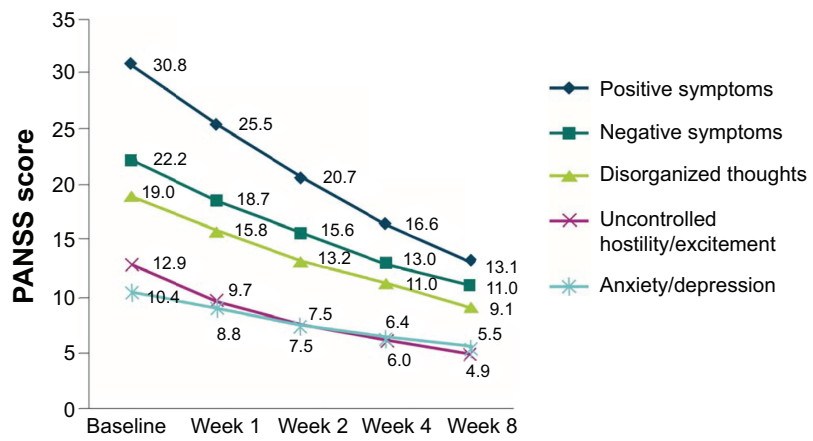

Figure 7 Mean PANSS Marder factor scores at each visit. Abbreviation: PANSS, Positive and Negative Syndrome Scale. 


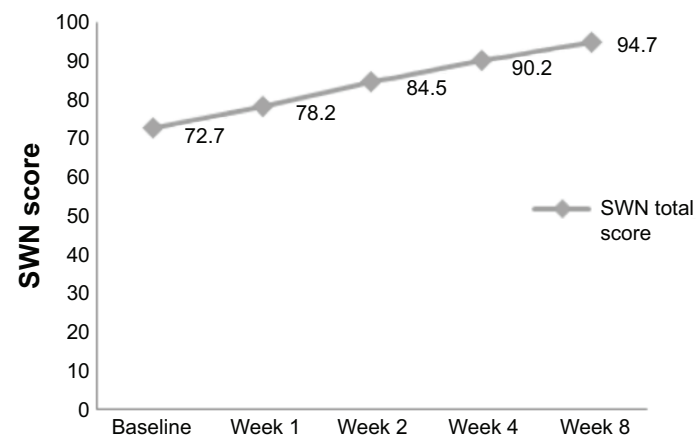

Figure 8 Mean SWN total score over time.

Abbreviation: SWN, Subjective Well-being under Neuroleptics.

points, 83 and 96, were defined, resulting in three categories (feeling discomfort [SWN $<83$ ], borderline [ $83 \leq \mathrm{SWN} \leq 96$ ], and feeling comfort [SWN >96]). ${ }^{16}$ The results of this study demonstrated overall improvement in mean SWN scores from baseline (72.7) to endpoint (94.7), with an increased percentage of patients indicating satisfaction with treatment (8.5\% to 56.9\%). As explored in one study, FEP patients with better improvement in SWN scores in early stage of treatment ( $\mathrm{SWN} \geq 80$ within the initial 2 weeks) could likely show a better efficacy in a short period and improved functioning outcome in the following 3-5 years. ${ }^{17}$ Improved SWN scores at week 2 in our study also suggest a good functional outcome with long-term pali-ER treatment.

Our study had a comparatively large sample size when compared with previous FEP studies. ${ }^{18,19}$ We also investigated the relationship between duration of untreated psychosis and scores on scales for social functioning (PANSS or PSP), which indicated that a shorter untreated period is associated with better social functioning (PSP score) and an improved PANSS score in patients with FEP once treatment is started. These findings are consistent with those of several other studies. ${ }^{5}$ The improvement in negative symptoms after pali-ER treatment is also consistent with the findings of a previous placebo-controlled study in a predominantly white population. ${ }^{9}$

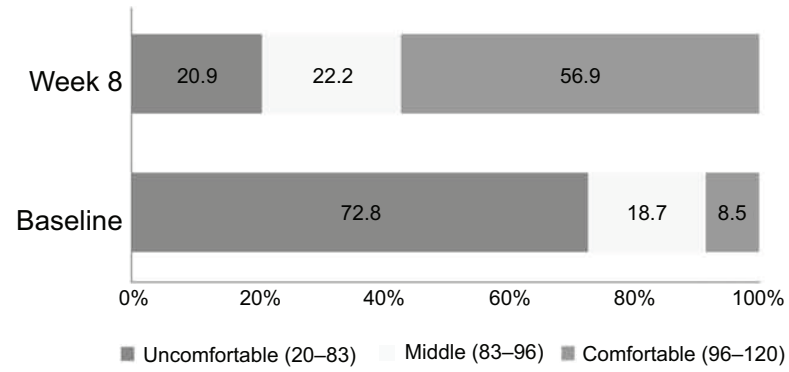

Figure 9 Subjective comfort level at baseline and endpoint.
Table 2 Incidence of treatment-emergent adverse events in at least $2 \%$ of patients (safety analysis set)

\begin{tabular}{llll}
\hline Adverse events & Patients & Frequency & Incidence (\%) \\
\hline Total & 95 & 225 & 30.8 \\
Extrapyramidal symptoms & 37 & 44 & 12.0 \\
Agitation & 13 & 14 & 4.2 \\
Somnolence & 13 & 13 & 4.2 \\
Dry mouth & 11 & 11 & 3.6 \\
Weakness & 11 & 11 & 3.6 \\
Bradykinesia & 10 & 10 & 3.3 \\
Dizziness & 8 & 9 & 2.6 \\
\hline
\end{tabular}

The safety and tolerability results for pali-ER over the course of the 8-week treatment period in patients with FEP are consistent with previous studies in a similar population ${ }^{12,13}$ and placebo-controlled trials conducted predominantly in white patients. ${ }^{20-22}$ The incidence of extrapyramidal TEAEs and increased prolactin levels in the current study was less than in other pali-ER studies conducted in the People's Republic of China ${ }^{23,24}$ and in pivotal global studies. ${ }^{8}$ This may reflect the low mean dose of pali-ER used in this study. However, the increase in body weight at study endpoint was consistent with that in previous pivotal studies.

This study has some limitations, including its open-label design and lack of an active or placebo comparator. The onset of effective treatment and dose required for improvement in psychotic symptoms and social functioning could have been better interpreted in comparison with an active or placebo comparator.

To summarize, flexibly dosed pali-ER treatment resulted in a significant improvement in psychotic symptoms and social functioning and was generally tolerable during this 8-week, open-label trial in Chinese patients with FEP.

\section{Acknowledgments}

The authors thank the study participants, without whom the study could not have been performed, and the following investigators for their participation: Yang Fude, Li Tao, Xu Yi, Zhang Kerang, Xu Xiufeng, Lv Luxian, Dong Jicheng, Yang Kongjun, Yang Shaohai, Tian Guoqiang, and Zheng Jingtao. They are grateful to Dr Guo Jiyuan, consultant psychiatrist, Beijing Min Kang Hospital, for his kind help in providing the SWN scale, his valuable comments, and excellent training on the SWN. The authors also thank Rishabh Pandey (SIRO Clinpharm Pvt Ltd) for writing assistance and Dr Wendy Battisti (Janssen Research and Development, LLC) for additional editorial assistance. 


\section{Author contributions}

TS, QR, and YW were involved in all aspects of the study design, study conduct, data interpretation, and manuscript composition. QT and KZ were involved in data interpretation and manuscript composition. All authors met ICMJE criteria and all those who fulfilled those criteria are listed as authors. All authors had access to the study data and made the final decision about where to present these data.

\section{Disclosure}

Xian-Janssen Pharmaceutical Ltd funded this study and was responsible for the study design and data collection, its analysis, and its interpretation. The sponsor was also responsible for deciding to publish the data and provided a formal review of the manuscript.

Data from this study were presented at the Ninth Psychiatric Forum of the West China Psychiatric Association in July 2012, the Seventh Annual Academic Conference of the Chinese Society of Psychiatry in October 2012, and at the Fourth Schizophrenia International Research Society Conference in April 2014.

TS was the principal investigator in the study, who is supported by research grants from the National Science and Technology Major Projects for "Major New Psychiatric Drugs Innovation and Development" (20112X09302-004). She has also received grant support from the National Natural Science Foundation of China (81171284). YW was employed by Janssen Research and Development during the study and QR is employed by Janssen Research and Development. The other authors have no conflicts of interest in this work.

\section{References}

1. Ran MS, Chen EY, Conwell Y, et al. Mortality in people with schizophrenia in rural China: 10-year cohort study. Br J Psychiatry. 2007;190: 237-242.

2. Xiang YT, Ma X, Cai ZJ, et al. Ungvari GS. Prevalence and sociodemographic correlates of schizophrenia in Beijing, China. Schizophr Res. 2008;102:270-277.

3. Li H, Rui Q, Ning X, Xu H, Gu NA. Comparative study of paliperidone palmitate and risperidone long-acting injectable therapy in schizophrenia. Prog Neuropsychopharmacol. 2011;35:1002-1008.

4. Prikryl R, Prikrylova-Kucerova H, Vrzalova M, Ceskova E. Role of long-acting injectable second-generation antipsychotics in the treatment of first-episode schizophrenia: a clinical perspective. Schizophr Res Treatment. 2012;2012:764-769.

5. Alvarez-Jiménez M, Priede A, Hetrick SE, McGorry PD, Gleeson JF. Preventing the second episode: a systematic review and meta-analysis of psychosocial and pharmacological trials in first-episode psychosis. Schizophr Bull. 2011;37:619-630.

6. Challis S, Nielssen O, Harris A, Large M. Systematic meta-analysis of the risk factors for deliberate self-harm before and after treatment for first-episode psychosis. Acta Psychiatr Scand. 2013;127:442-454.
7. Gleeson JF, Alvarez-Jimenez M, Lederman R. Moderated online social therapy for recovery from early psychosis. Psychiatr Serv. 2012;63:719.

8. Meltzer HY, Bobo WV, Nuamah IF, et al. Efficacy and tolerability of oral paliperidone extended-release tablets in the treatment of acute schizophrenia: pooled data from three 6-week, placebo-controlled studies. J Clin Psychiatry. 2008;69:817-829.

9. Canuso CM, Bossie CA, Turkoz I, Alphs L. Paliperidone extended-release for schizophrenia: effects on symptoms and functioning in acutely ill patients with negative symptoms. Schizophr Res. 2009;113:56-64.

10. Guoxing C, Guojian X. Observation of efficacy and safety of paliperidone sustained release tablets and domestic olanzapine in treatment of schizophrenia. Chongqing Med. 2011;17:13.

11. Zhang Y, Dai G. Efficacy and metabolic influence of paliperidone ER, aripiprazole and ziprasidone to patients with first-episode schizophrenia through 52 weeks follow-up in China. Hum Psychopharmacol. 2012;27: 605-614.

12. Yu YM, Li HF, Yang PD. Efficay, safety and toleriability of paliperidone ER treatment on acute schizophrenia at flexible dosage. Shanghai Jingshen Yixue. 2010;22:349-353.

13. Sun L, Zhou T, Lei T. Efficacy and safety of paliperidone ER in the treatment of adolescent patients with first-episode schizophrenia. Chinese J New Drugs. 2011;9:009.

14. Shu L. Chinese Guideline for Schizophrenia. Beijing, People's Republic of China: Peking University Medical College Press; 2007.

15. Guo J, Zhao Z, Ha S. Testing the validity and reliability of Chinese version of Subjective Well-Being under Neuroleptics (SWN) Short Form. Med J Chinese People Health. 2003;15:705-707.

16. Guo J, Tian F, Jia H. An analysis of the tested results for 126 schizophrenic patients by Chinese version of Subjective Well-Being under Neuroleptics (SWN) Short Form. Med J Chinese People Health. 2003; 15:513-515.

17. de Haan L, Weisfelt M, Dingemans PM, Linszen DH, Wouters L. Psychometric properties of the Subjective Well-Being Under Neuroleptics scale and the Subjective Deficit Syndrome Scale. J Psychopharmacol. 2002; 162:24-28.

18. Huq ZU. A trial of low doses of risperidone in the treatment of patients with first-episode schizophrenia, schizophreniform disorder, or schizoaffective disorder. J Clin Psychopharmacol. 2004;24:220-224.

19. Crespo-Facorro B, Perez-Iglesias R, Ramirez-Bonilla M, MartinezGarcia O, Lorca J, Vazquez-Barquero JL. A practical clinical trial comparing haloperidol, risperidone, and olanzapine for the acute treatment of first-episode nonaffective psychosis. J Clin Psychiatry. 2006; 67:1511-1521.

20. Davidson M, Emsley R, Kramer M, Ford L, Pan G, Lim P. Efficacy, safety and early response of paliperidone extended-release tablets (paliperidone ER): results of a 6-week, randomized, placebo-controlled study. Schizophr Res. 2007;93:117-130.

21. Kane J, Canas F, Kramer M, et al. Treatment of schizophrenia with paliperidone extended-release tablets: a 6-week placebo-controlled trial. Schizophr Res. 2007;90:147-161.

22. Emsley R, Berwaerts J, Eerdekens M, et al. Efficacy and safety of oral paliperidone extended-release tablets in the treatment of acute schizophrenia: pooled data from three 52-week open-label studies. Int Clin Psychopharmacol. 2008;23:343-356.

23. Lieberman JA, Koreen AR, Chakos M, et al. Factors influencing treatment response and outcome of first-episode schizophrenia: implications for understanding the pathophysiology of schizophrenia. J Clin Psychiatry. 1996;57:5-9.

24. Hong-Yan Z, Xiao-Nan H, Xue-Qin W. An open-label study on the safety and efficacy of paliperidone extended-release in non-acute schizophrenic patients. Chinese J Psychiatry. 2012;45. 


\section{Supplementary material}

Table SI Enrollment and analysis sets for all sites

\begin{tabular}{|c|c|c|c|c|}
\hline Site & Enrollment & FAS & PPS & SS \\
\hline Sixth Hospital of Peking University & 12 & 8 & 5 & 9 \\
\hline Xijing Hospital of the Fourth Military Medical University & 19 & 19 & 15 & 19 \\
\hline First Hospital of ShanXi Medical University & 27 & 26 & 23 & 26 \\
\hline Psychiatric Hospital of HeNan Province & 12 & 12 & 12 & 12 \\
\hline Mental Health Center of QingDao City & 67 & 67 & 67 & 67 \\
\hline KangNing Hospital of ShenZhen City & 12 & 12 & 5 & 12 \\
\hline KangNing Hospital of WenZhou City & 84 & 82 & 67 & 83 \\
\hline The Seventh People's Hospital & 24 & 24 & 22 & 24 \\
\hline \multicolumn{5}{|l|}{ of ShaoXing City } \\
\hline The Fourth Hospital of FuZhou City & 56 & 56 & 53 & 56 \\
\hline Total & 313 & 306 & 269 & 308 \\
\hline
\end{tabular}

Abbreviations: FAS, full analysis set; PPS, per-protocol set; SS, safety set.

\section{Publish your work in this journal}

Neuropsychiatric Disease and Treatment is an international, peerreviewed journal of clinical therapeutics and pharmacology focusing on concise rapid reporting of clinical or pre-clinical studies on a range of neuropsychiatric and neurological disorders. This journal is indexed on PubMed Central, the 'PsycINFO' database and CAS, and is the official journal of The International Neuropsychiatric Association (INA). The manuscript management system is completely online and includes a very quick and fair peer-review system, which is all easy to use. Visit http://www.dovepress.com/testimonials.php to read real quotes from published authors.

\footnotetext{
Submit your manuscript here: http://www.dovepress.com/neuropsychiatric-disease-and-treatment-journal
} 